\title{
Degradation of Methacrylate Monomers in Human Saliva
}

\author{
Maki HAGIO ${ }^{1}$, Minoru KAWAGUCHI ${ }^{2}$, Wataru MOTOKAWA ${ }^{1}$ and Koji MIYAZAKI ${ }^{2}$ \\ ${ }^{1}$ Department of Oral Growth \& Development, Fukuoka Dental College, 2-15-1 Tamura, Sawara-ku, Fukuoka 814-0193, Japan \\ ${ }^{2}$ Department of Dental Engineering, Fukuoka Dental College, 2-15-1 Tamura, Sawara-ku, Fukuoka 814-0193, Japan \\ Corresponding author, Maki Hagio E-mail:makihagio@college.fdcnet.ac.jp
}

Received December 8, 2005/Accepted January 25, 2006

\begin{abstract}
This study assessed the effect of the molecular structure of newly synthesized methacrylate monomers on their chemical stability in human saliva, whereby these monomers can be used as dental composite resins. Six model monomethacrylates and two urethane-modified BisGMA monomers were added to human saliva, and their change in concentration after 24 , 48, and 72 hours were measured by high-performance liquid chromatography. Degradation of the six model monomethacrylate monomers was found to be influenced by the molecular structure, such as steric hindrance and presence of urethane bond in chemical backbone. Based on the degradation test results of these six monomers, urethane-modified BisGMA derivatives - in which the hydroxyl groups in original BisGMA monomer were substituted with alkyl isocyanate - were synthesized and subjected to degradation test. The urethane-modified BisGMA monomers showed a particular resistance to salivary hydrolysis. Results of this study thus suggested that urethane groups should be considered when designing new monomers for dental composite systems as they demonstrated improved resistance to hydrolysis.
\end{abstract}

Key words: Monomer, Degradation, Saliva

\section{INTRODUCTION}

Degradation of dental resins in human saliva, that lead to decrease in mechanical strength and surface hardness, have been reported in in vitro studies ${ }^{1,2}$. The results of these studies suggested that such degradation arose from the chemical degradation of methacrylate polymers due to enzyme-catalyzed hydrolysis reaction of ester bond. However, methods that can adequately investigate the enzymatic degradation mechanism involved in the breakdown of methacrylate polymers are not available. Therefore, the chemical degradation of methacrylate monomers has been a subject of research to clarify the hydrolytic degradation behavior of ester linkages in polymer networks ${ }^{3-5)}$.

Several studies have been conducted to reveal the degradation behavior of bisphenol A diglycidyl dimethacrylate (BisGMA) and triethylenglycol dimethacrylate (TEGDMA) monomers in phosphate buffer solution containing cholesterol esterase which simulates salivary enzyme solution ${ }^{6,7)}$. The hydrolyzed products of these monomers were detected in the simulated solution by enzymatic attack. The results of these studies revealed that a rapid hydrolysis reaction with the enzyme occurred within several days. It is known that esterase, a salivary enzyme, displays structure-dependent hydrolytic activity in the hydrolysis of esters such as fatty acid ester $^{8)}$. However, there is a lack of information regarding the relationship between the chemical structure of methacrylate monomers and the hydrolysis by salivary enzymes. It is expected that the develop- ment of a new methacrylate monomer with higher resistance to hydrolysis by salivary enzymes would contribute to improving the durability of composite resins in vivo.

Recently, Jaffer et $a l .^{9)}$ measured the chemical stability of dimethacrylate monomers in human saliva and revealed that the degradation of BisGMA was faster than that of urethane methacrylate oligomer. The findings of this study should serve to expedite research activities that focus on how the structural dependency of methacrylate monomers influence their resistance to hydrolysis. In particular, BisGMA is the most common dimethacrylate monomer of composite resin materials ${ }^{10,11}$. Therefore, improvement in the chemical stability of BisGMA in human saliva would result in enhanced biostability of composite resin restorations.

Jaffer et $a l .{ }^{9)}$ had demonstrated that the human saliva contains sufficient esterase activity to catalyze the degradation of monomers. The aim of this study, therefore, was to assess the chemical stability of methacrylate monomers in human saliva to reveal the relationship between enzymatic degradation resistance and the chemical structure of monomers. Further, to examine the stability of the experimental monomers under clinical conditions, degradation test was carried out in human whole saliva. Six types of model monomethacrylate monomer and two BisGMAderivative monomers were synthesized, and their degradation as a function of time was investigated by using high-performance liquid chromatography (HPLC). 


\section{MATERIALS AND METHODS}

Preparation of model monomethacrylate and BisGMAderivative monomers

All chemicals used were purchased from Aldrich (Milwaukee, WI, USA) and Tokyo Kasei (Tokyo, Japan), and used as-received. BisGMA and UDMA were obtained from commercial products (ShinNakamura Chemical, Wakayama and Nemoto Chemical, Ishikawa, Japan). Chemical structures of six model monomethacrylates and two BisGMA-derivative monomers were shown in Figs. 1 and 2 respectively. 2-hydroxy-3-methacryloyloxypropoxyphenyl (HPPM) was synthesized by the reaction of $0.3 \mathrm{~mol}$ of methacrylic acid with 0.2 mol of phenyl glycidyl ether in the presence of triethylammonium chloride, and then purified as described ${ }^{12,13)}$. 2-methacryloyloxyethylphenyl (PEMA), 4-methacryloyloxybutylphenyl (PBMA) and 1-phenyl-2-methacryloyloxybutane (PEEM) were prepared by the reaction of methacryloyl chloride with the corresponding phenyl alkyl alcohol. For example in the case of PEMA, 2phenylethanol $(0.2 \mathrm{~mol})$ and triethylamine $(0.2 \mathrm{~mol})$ were dissolved in $200 \mathrm{ml}$ of ethyl ether. Methacryloyl chloride $(0.2 \mathrm{~mol})$ was added dropwise with stirring. After four hours, the mixture was filtrated and evaporated. The molecular structure of obtained liquid was confirmed by NMR and FT-IR.

3-methacryloyloxyethoxycarbonylaminopropane

(MCI-Pr) and 2-methacryloyloxyethoxycarbonylaminoethyoxyphenyl (MCI-PE) were prepared according to the following method. $0.2 \mathrm{~mol}$ of propanol or 2-phenoxylethanol and $0.2 \mathrm{~mol}$ of 2 -

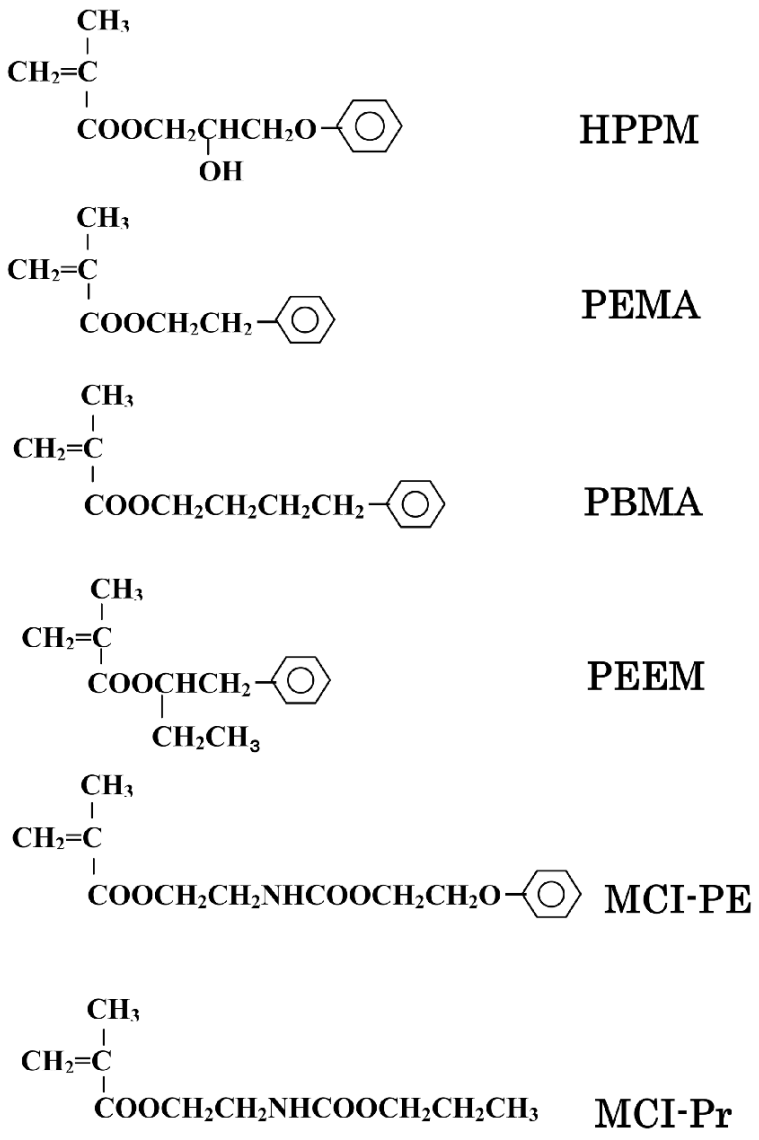

Fig. 1 Chemical structures of model monomethacrylates.<smiles>C=C(C)C(=O)OCC(COc1ccc(C(C)(C)c2ccc(OCC(COC(=O)C(C)(C)C)OC(=O)NCCC)cc2)cc1)C(=O)NCCC</smiles>

BisGMA-Pr<smiles>C=C(C)C(=O)OCC(COc1ccc(C(C)(C)c2ccc(OCC(COC(=O)C(=C)C)C(=O)NCCCC)cc2)cc1)OC(=O)NCCCC</smiles>

\section{BisGMA-Bu}

Fig. 2 Chemical structures of BisGMA-derivative monomers. 
methacryloyloxyethyl isocyanate (Showa Denko, Kawasaki, Kanagawa) were dissolved in $200 \mathrm{ml}$ of dry dichloromethane. Dibutyltin dilaurate $\left(10^{-4} \mathrm{~mol}\right)$ was added to the reaction mixture under reflux (approximately $40^{\circ} \mathrm{C}$ ) for three hours. After solvent removal by evaporation under vacuum, a clear viscous liquid was obtained. Two BisGMA-derivative monomers, 2,2-bis (2-propoxycarbonylamino3-methacryloyloxypropoxyphenyl) propane (BisGMAPr) and 2,2-bis (2-buthoxycarbonylamino-3methacryloyloxypropoxyphenyl) butane (BisGMA$\mathrm{Bu})$, were synthesized by the reaction of BisGMA $(0.3 \mathrm{~mol})$ and alkyl isocyanate (propyl isocyanate or butyl isocyanate: $0.6 \mathrm{~mol}$ ) in the presence of dibutyltin dilaurate in dichloromethane as the method described above. The molecular structure of obtained liquid was confirmed by NMR and FT-IR.

\section{Preparation of human saliva}

Human whole saliva was collected into $50-\mathrm{ml}$ polyethylene centrifuge tube from eight healthy individuals and immediately stored on ice before being processed according to a previously reported method ${ }^{9)}$. All procedures were conducted according to human ethics protocol approved by the Fukuoka Dental College. Bulk debris was separated from whole saliva by centrifugation at $2400 \mathrm{rpm}$ for 10 minutes at $4^{\circ} \mathrm{C}$. The supernatant was collected and then filtered through a $0.22 \mu \mathrm{m}$ PTFE syringe filter (Steriflip, Millipor, Bedford, MA, USA). Each filtrate was collected and stored in a sterilized glass bottle. Saliva samples $(\mathrm{pH} 7.3)$ were then stored in a refrigerator $\left(-20^{\circ} \mathrm{C}\right)$ before use.

\section{Degradation test}

Degradation test was carried out with two different concentrations (5 and $10 \mathrm{mM}$ ) for each monomethacrylate monomer. The concentration range chosen in this study was determined based on data from preliminary experiments, where detectable degradation was observed in this optimum concentration range for all synthesized monomers. Each monomer was diluted with DMSO (Wako, Osaka, Japan) to the above-mentioned concentrations. After which, $100 \mu \mathrm{l}$ of each solution was added to $3 \mathrm{ml}$ of human saliva in glass tubes such that the 5 and $10 \mathrm{mM}$ concentrations in human saliva yielded the final concentrations of 0.161 and $0.322 \mathrm{mM}$ respectively. All sample solutions were stored with horizontal shaking (80 cycles per min) at $37^{\circ} \mathrm{C}$ for three days. After incubation for 24,48 , and 72 hours, $20 \mu$ l of sample solution was removed with a microsyringe and subjected to HPLC analysis.

HPLC analyses were carried out using Toso HPCL system (Tokyo, Japan) with an ODS column (grain size: $5 \mu \mathrm{m}$, column size: $4.6 \phi \times 250 \mathrm{~mm}$, TSK, Toso, Tokyo, Japan) at a flow rate of $1 \mathrm{ml} / \mathrm{min}$. Mobile phase was acetonitrile-water $(70: 30 \mathrm{v} / \mathrm{v})$ and the monitoring wavelength was $230 \mathrm{~nm}$ (UV-8011, Toso, Tokyo, Japan). For aliphatic methacrylate monomer, MCI-Pr, monitoring wavelength was set to $210 \mathrm{~nm}$. All experiments were performed in triplicate.

Brinell hardness measurement of resins based on BisGMA-derivative monomers

For the two urethane-modified BisGMA monomers, the Brinell hardness $\left(\mathrm{H}_{\mathrm{B}}\right)$ values of their cured materials were measured. For control, a monomer mixture of urethane-modified BisGMA and TEGDMA with a ratio of $50 / 50$ (wt $\%$ ) containing photoinitiator $(0.5 \mathrm{wt} \%$ of camphoroquinone and 1.0 wt\% of 2-dimethylaminoethyl methacrylate, both reagents were obtained from Tokyo Kasei, Tokyo, Japan) was poured into a Teflon ${ }^{\circledR}$ mold $(10 \mathrm{~mm}$ of inner diameter and $3 \mathrm{~mm}$ of depth) and cured with a light-curing unit (90-second exposure, Dentacolor XL-S, Kulzer, Germany). Hardness measurement was carried out by Brinell hardness tester (Mori, Tokyo, Japan) with $30 \mathrm{~kg}$ load $(n=5)$.

\section{Statistical analysis}

For the degradation of monomers, a Scheffe's multiple comparison test after two-way analysis of variance was applied for each experiment. Statistical significance for all measurements was set at $\mathrm{p}<0.05$.

\section{RESULTS}

Degradation of methacrylate monomers in human saliva

Degradation of model monomethacrylate monomers in human saliva as a function of time was illustrated in Figs. 3 and 4. Each graph was shown in the percentage of change in concentration to the initial concentration. As shown in Fig. 3, for model monomers containing phenyl alkyl backbone - namely HPPM, PEMA, and PBMA, they showed significantly higher decrease in concentration with increasing incubation time than PEEM. After 72-hour incubation, the final concentration of HPPM, PEMA, and PBMA ranged from 0 to $15 \%$. The decreasing rate of these three monomers was in the order of PBMA $>$ HPPM $>$ PEMA. On the other hand, PEEM showed the least decrease in concentration and remained at $65 \%$ of initial concentration after 72-hour incubation.

For MCI-PE and MCI-Pr, these monomers contained a urethane bond in their chemical structures. They showed less degradation than alkyl monomers with a straight alkyl chain and an aromatic ring at its end. After 72-hour incubation, the concentrations of MCI-PE and MCI-Pr remained at 70 and 55\% respectively.

Degradation test results for $10 \mathrm{mM}$ of monomer concentration (Fig.4) showed a similar tendency as that of $5 \mathrm{mM}$. However, the final concentration of 


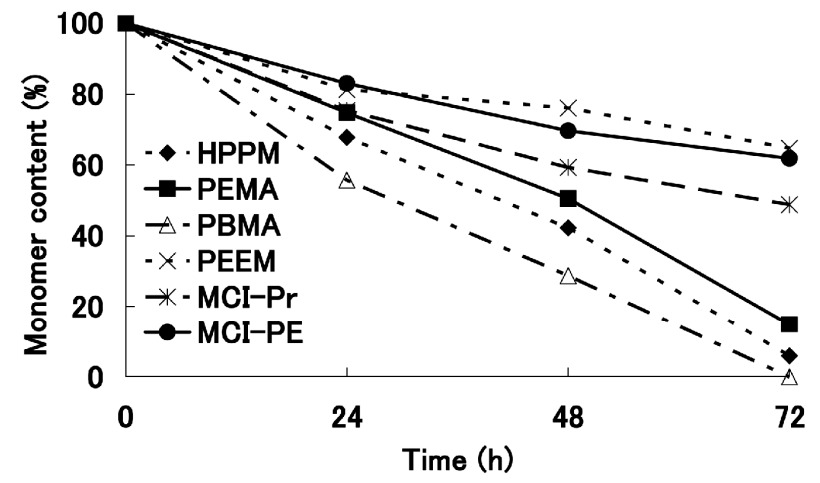

Fig. 3 Reduction rate of model monomethacrylates (initial conc: $5 \mathrm{mM}$ ).

each monomer tested at $10 \mathrm{mM}$ was significantly higher than that at $5 \mathrm{mM}$. After 72-hour incubation, the concentration of phenyl alkyl methacrylates (except for PEEM) remained at 22-31\%, while that of urethane bond-containing monomers remained at $55-70 \%$.

Fig. 5 shows the degradation of two urethanemodified BisGMA monomers as well as a conventional BisGMA and UDMA in human saliva. While the conventional BisGMA showed a rapid decrease in concentration within the initial 24 hours and degraded completely after 48 hours' incubation, UDMA - which possess urethane bonds - showed a moderate decrease with increasing incubation time. After 72 -hour incubation, approximately $70 \%$ of the initial amount of UDMA remained. In contrast, for urethane-modified monomers BisGMA-Pr and BisGMA$\mathrm{Bu}$, there were no significant changes in concentration throughout the 72-hour incubation period. Hence, these two urethane-modified BisGMA monomers showed a superior stability in human saliva as compared to BisGMA and UDMA monomers.

\section{Brinell hardness measurement}

Table 1 shows the Brinell hardness values of the resins. The resin based on BisGMA showed a greater hardness $\left(\mathrm{H}_{\mathrm{B}}\right.$ : 31.6) than urethane-modified BisGMAbased resins.

\section{DISCUSSION}

The results of this study clearly showed that the degradation resistance of model monomethacrylate monomers by enzymatic attack was dependent on the chemical structure of monomers. The degradation test for phenyl alkyl methacrylate monomers with straight alkyl chain revealed that the monomer with shorter carbon chain length (PEMA: ethylene chain) showed higher stability than the monomer with longer carbon chain length (PBMA: butylene chain). On this note, it may be possible that there was a structural correlation between carbon length of alkyl

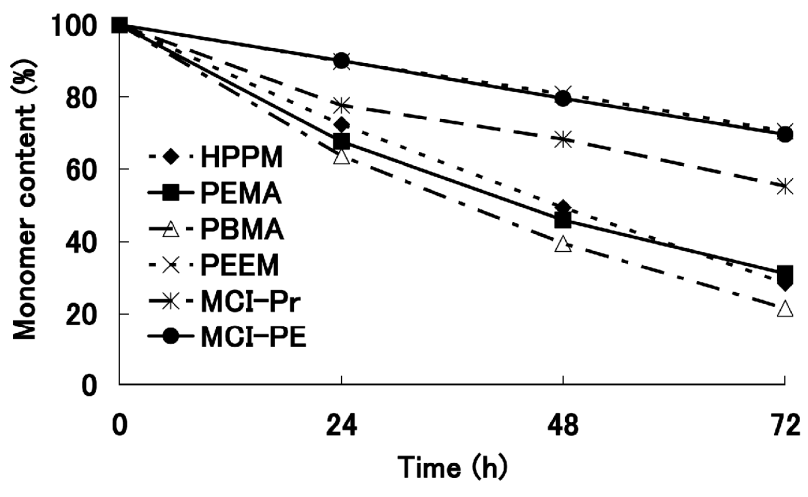

Fig. 4 Reduction rate of model monomethacrylates (initial conc: $10 \mathrm{mM}$ ).

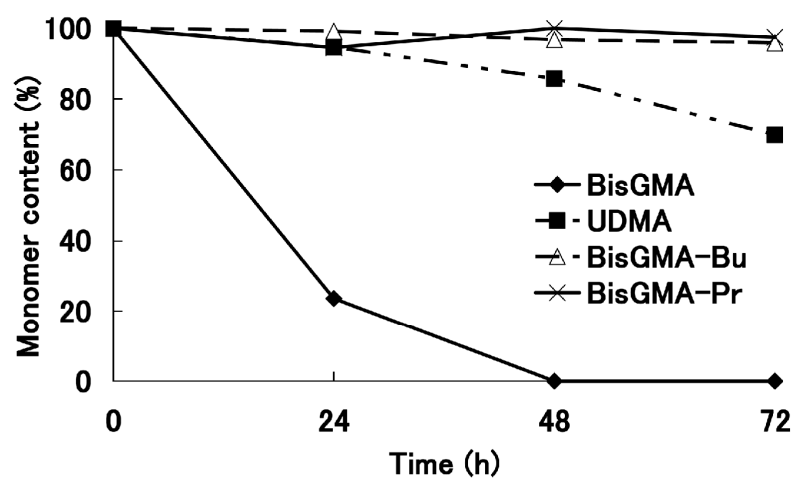

Fig. 5 Reduction rate of urethane-modified BisGMAderivative monomers (initial conc: $10 \mathrm{~m} \mathrm{M}$ ).

Table 1 Brinell hardness of resins based on BisGMA and urethane-modified BisGMA monomers

\begin{tabular}{cccc}
\hline & BisGMA & BisGMA-Pr & BisGMA-Bu \\
\hline $\mathrm{H}_{\mathrm{B}}$ & 31.58 & $26.72^{*}$ & $25.65^{*}$ \\
$\mathrm{SD}$ & 0.02 & 0.05 & 0.05 \\
\hline
\end{tabular}

*not significant $(\mathrm{p}>0.05)$

chain and degradation resistance. However, the reason for lower stability against the degradation of longer carbon chain lengths is not clear. Concerning the effect of hydroxyl group on the chemical stability of monomers such as HPPM, there was no distinct impact on degradation behavior as compared with PEMA and PBMA. However, it seemed that the branched alkyl chain - such as the ethylene side chain of PEEM - had a potential to improve degradation resistance. If this were so, then it would be due to the steric hindrance of branched alkyl backbone against hydrolysis ${ }^{14)}$.

For monomethacrylate monomers with a urethane bond in the backbone, namely MCI-Pr and MCI-PE, they showed no degradation when compared with the other monomethacrylate monomers (HPPM, PEMA, and PBMA) - except PEEM. It seemed 
that the presence of urethane bond increased their chemical stability in human saliva. Pierre and Chiellini ${ }^{15)}$ reported that the presence of urethane structure within polymeric materials could reduce the susceptibility to hydrolysis. In their report, the urethane bond was shown to contribute to the formation of intermolecular hydrogen-bonded structures, thereby minimizing the enzyme's access to the cleavage sites of urethane polymers.

Considering the enhanced stability imparted by urethane group as seen in MCI-Pr and MCI-PE, we therefore designed urethane-modified BisGMA monomers (BisGMA-Pr and BisGMA-Bu) and evaluated their chemical stability in human saliva. Indeed, the chemical stability of BisGMA-Pr and BisGMA-Bu in human saliva were superior to conventional BisGMA monomer. To prepare BisGMA-Pr and BisGMA-Bu, two hydroxyl groups on BisGMA underwent reaction with alkyl isocyanate. The pendant alkyl urethane substituents of the urethane-modified BisGMA monomers then formed intramolecular hydrogen bond ( $\mathrm{NH}$ of urethane and $\mathrm{C}=\mathrm{O}$ of ester) and caused structural incompatibility against enzymatic attack. Therefore, the higher resistance to hydrolytic degradation of these urethane-modified BisGMA monomers would be due to both the intra- and intermolecular hydrogen-bonded structures. In terms of Brinell hardness measurement, the urethane-modified BisGMA monomers showed slightly lower hardness than BisGMA. Nevertheless, these urethane-modified BisGMA monomers' superior resistance to enzymatic degradation would result in stable mechanical properties in vivo.

Several researchers demonstrated that in human saliva, the enzymatic activity of hydrolase reaches levels high enough to degrade composite resin monomers ${ }^{16,17)}$. In the present study, the modified BisGMA monomers were shown to have a higher resistance to enzymatic hydrolysis as compared with conventional BisGMA monomer. Hence, the substitution of hydroxyl groups of BisGMA with alkyl urethane linkage favorably contributed to the overall chemical stability of the modified BisGMA-based materials. These urethane-modified BisGMA monomers thus have the potential as dental monomer systems that can improve the chemical stability of dental composite materials.

In conclusion, the resistance to degradation of model monomethacrylate monomers in human saliva was dependent on their molecular structure. Introduction of urethane linkage and branched alkyl group to the carbon chain backbone of monomethacrylates could improve the latter's degradation resistance. In the light of these findings, urethane-modified BisGMA monomers could be utilized to develop new dental composites with better chemical stability compared to similar composites that employ conventional BisGMA as a base monomer.
However, as the toxicity of hydrolyzed products is still unknown, we would recommend further studies on the biocompatibility of hydrolyzed products.

\section{REFERENCES}

1) Munksgaard EC, Freund M. Enzymatic hydrolysis of (di) methacrylates and their polymers. Scand J Dent Res 1990; 98: 261-267.

2) Larsen IB, Munksgaard EC. Effect of human saliva on surface degradation of composite resins. Scand J Dent Res 1991; 99: 254-261.

3) Bean TA, Zhuang WC, Tong PY, Eick JD, Yourtee DM. Effect of esterase on methacrylates and methacrylate polymers in an enzyme simulator for biodurability and biocompatibility testing. J Biomed Mater Res 1994; 28: 59-63.

4) Santerre, JP, Shajii L, Leung BW. Relation of dental composite formulations to their degradation and the release of hydrolyzed polymeric-resin-derived products. Crit Rev Oral Biol Med 2001; 12: 136-151.

5) Freund M, Munksgaard EC. Enzymatic degradation of BISGMA/TEGDMA-polymers causing decreased microhardness and greater wear in vivo. Scand J Dent Res 1990; 98: 351-355.

6) Yourtee DM, Smith RE, Russo KA, Burmaster S, Cannon JM, Eick JD, Kostoryz EL. The stability of methacrylate biomaterials when enzyme challenges: Kinetic and systematic evaluations. J Biomed Mater Res 2001; 57: 522-531.

7) Finer Y, Santerre JP. The influence of resin chemistry on a dental composite's biodegradation. J Biomed Mater Res 2004; 69A: 233-246.

8) Chauncey HH. Salivary enzymes. JADA 1961; 63: 360368.

9) Jaffer F, Finer Y, Santerre JP. Interactions between resin monomers and commercial composite resins with human saliva derived esterases. Biomaterials 2002; 23: 1707-1719.

10) Lovell LG, Newman SM, Bowman CN. The effects of light intensity, temperature, and comonomer composition on the polymerization behavior of dimethacrylate dental resins. J Dent Res 1999; 78: 1469-1476.

11) Sideridou I, Tserki V, Papanastaiou G. Effect of chemical structure on degree of conversion in lightcured dimethacrylate-based dental resins. Biomaterials 2002; 23: 1819-1829.

12) Brzozowski ZK, Nowicki A. Unsaturated resins obtained from bisphenols and glycidyl esters of acrylic acids. J Polym Sci, Polym Chem Ed 1975; 13: 14781479.

13) Kawaguchi M, Fukushima T, Horibe T. Effect of monomer structure on the mechanical properties of light-cured unfilled resins. Dent Mater J 1988; 7: 174181.

14) Moszner N, Salz U, Zimmermann J. Chemical aspects of self-etching enamel-dentin adhesives: A systematic review. Dent Mater 2005; 21: 895-910.

15) Pierre T, Chiellini E. Biodegradability of synthetic polymers used for medical and pharmaceutical applica- 
tions. Part 1: Principles of hydrolysis mechanisms. J Bioact Compat Polym 1986; 1: 467-497.

16) Finer Y, Santerre JP. Salivary esterase activity and its association with the biodegradation of dental composite. J Dent Res 2004; 83: 22-26.
17) Lin BA, Jaffer F, Duff MD, Tang YW, Santerre JP. Identifying enzyme activities within human saliva which are relevant to dental resin composite biodegradation. Biomaterials 2005; 26: 4259-4264. 